\title{
Entropy of Bogoliubov Automorphisms of the Canonical Anticommutation Relations
}

\author{
Erling St $\phi$ rmer $^{1}$ and Dan Voiculescu ${ }^{2}$ \\ 1 Department of Mathematics, University of Oslo, N-0316 Oslo 3, Norway \\ 2 Department of Mathematics, University of California, Berkeley, Berkeley, CA 94720, USA
}

Received March 1, 1990

\begin{abstract}
We compute the entropy $h_{\omega_{A}}\left(\alpha_{U}\right)$ in the sense of Connes, Narnhofer and Thirring of Bogoliubov automorphisms $\alpha_{U}$ of the CAR-algebra with respect to invariant quasifree states $\omega_{A}$ with $0 \leqq A \leqq 1$ having pure point spectrum.
\end{abstract}

\section{Introduction}

In their recent paper [3] Connes, Narnhofer, and Thirring extended the definition of entropy for automorphisms of finite von Neumann algebras studied in [4] to the case of automorphisms of $C^{*}$-algebras invariant with respect to a given state. In the present paper we shall compute this for Bogoliubov automorphisms of the CAR-algebra with respect to invariant quasifree states. Recall, for more details see Sect. 4, that if $H$ is a complex Hilbert space and $f \rightarrow a(f)$ is a representation of $H$ in the CAR-algebra $\mathscr{A}(H)$ satisfying the canonical anticommutation relations then each unitary operator $U$ on $H$ defines a Bogoliubov automorphism $\alpha_{U}$ of $\mathscr{A}(H)$ by $\alpha_{U}(a(f))=a(U f)$. If $A \in B(H)$ satisfies $0 \leqq A \leqq 1$ and $A U=U A$, then $\alpha_{U}$ is invariant with respect to the (gauge invariant) quasifree state $\omega_{A}$ defined by $A$. In the case $A=\frac{1}{2} 1$, i.e. $\omega_{A}$ is the unique tracial state $\tau$ on $\mathscr{A}(H)$, then the entropy $h_{\tau}\left(\alpha_{U}\right)$ is the same as that of the extension of $\alpha_{U}$ to the GNS-representation of $\tau$ as defined in [4]. A. Connes suggested to us that the formula for the entropy should be

$$
h_{\tau}\left(\alpha_{U}\right)=\frac{\log 2}{2 \pi} \int_{0}^{2 \pi} m(U)(\theta) d \theta
$$

where $m(U)$ is the multiplicity function of the absolutely continuous part $U_{a}$ of $U$, a conjecture which initiated the present work. More generally, if $U_{a}$ acts on the

* Supported in part by a grant from the National Science Foundation 
subspace $H_{a}$ of $H$ we can write $H_{a}$ as a direct integral

$$
H_{a}=\int_{\mathbf{T}}^{\oplus} H_{\theta} d \theta
$$

with $d \theta$ the Lebesgue measure on the unit circle $\mathbf{T}$. Correspondingly, the operator $A$ commuting with $U$ decomposes on $H_{a}$ by the formula

$$
A_{a}=A \mid H_{a}=\int_{\mathrm{T}}^{\oplus} A(\theta) d \theta .
$$

Let $\eta$ denote the real function on $[0,1]$ defined by $\eta(0)=0, \eta(t)=-t \log t, t \in(0,1]$. Then if $A$ has pure point spectrum we shall prove the formula

$$
h_{\omega_{A}}\left(\alpha_{U}\right)=\frac{1}{2 \pi} \int_{0}^{2 \pi} \operatorname{Tr}(\eta(A(\theta))+\eta(1-A(\theta))) d \theta
$$

where $\operatorname{Tr}$ denotes the usual trace on $B\left(H_{\theta}\right)$.

For general $A$ we leave it as an open problem whether (2) is true. It is implicit in (2) that the entropy is unaffected by the singular part of $U$. We shall in particular show that if the spectral measure of $U$ is singular with respect to Lebesgue measure than $h_{\varphi}\left(\alpha_{U}\right)=0$ for all $\alpha_{U}$-invariant states $\varphi$.

In addition to giving a formula for the entropy of a large class of automorphisms and invariant states (2) also yields an example of entropy in a more technically complicated situation than in previous calculations $[1,2,3,4,7]$. Namely in those cases the computation is based on the existence of a natural maximal abelian subalgebra which is globally invariant under the automorphism. In the case of Bogoliubov automorphisms we cannot in general expect this.

The proof of (2) is divided into five sections. The first, Sect. 2, contains a characterization of the Lebesgue integral on the circle by its properties on a class of functions which in our applications will be multiplicity functions of unitary operators with Lebesgue spectral measures. In Sect. 3 we prove some basic general results on entropy that will be needed in the sequel following closely the theory developed in [3]. In Sect. 4 we study the canonical anticommutation relations in more detail and develop the basic techniques on entropy in the case of quasifree states and Bogoliubov automorphisms. In Sect. 5 we consider the case when the spectral measure of $U$ has nonzero singular part. In particular we show (2) in the simple case when the multiplicity function of the Lebesgue part of $U$ is constant on a finite number of arcs of rational length. Then the proof is completed in Sect. 6, first for the case when $A$ is a scalar operator, in which case the characterization in Sect. 2 is used, and then in the general case.

\section{Lebesgue Measure on the Circle}

In this section we show a result on the Lebesgue measure on the circle, which will be used to give the formula for the entropy of Bogoliubov automorphisms with respect to quasifree states defined by scalar operators.

Let $\mathscr{C}$ be the additive semigroup of functions $f: \mathbf{T} \rightarrow\{0\} \cup \mathbb{N}$ which are measurable with respect to Lebesgue measure $d \theta$ on $\mathbf{T}=\{z \in \mathbf{C}:|z|=1\}$. For further use we denote by $\mathbb{1}$ the constant function equal to 1 to $\mathbf{T}$ and by $T_{n}: \mathscr{C} \rightarrow \mathscr{C}(n \in \mathbb{N})$ 
the map

$$
\left(T_{n} f\right)(\rho)=\sum_{\substack{z \in \mathbf{T} \\ z^{n}=\rho}} f(z) .
$$

Let $\mu: \mathscr{C} \rightarrow \mathbb{R}^{+}$- the nonnegative reals - which satisfies the following conditions:

(i) $\mu(n \mathbb{1})=n, n \in\{0\} \cup \mathbb{N}$.

(ii) $f \leqq g \Rightarrow \mu(f) \leqq \mu(g)$.

(iii) $f_{j} \nearrow f \Rightarrow \mu\left(f_{j}\right) \nearrow \mu(f), j \in \mathbb{N}$.

(iv) $\mu\left(T_{n} f\right)=n \mu(f)$.

(v) $\mu(f)=\mu(g)$ if $f$ and $g$ are equal a.e. (with respect to Lebesgue measure).

In our applications $\mathscr{C}$ will consist of multiplicity functions of unitaries and $\mu$ will be a scalar multiple of the entropy of the corresponding Bogoliubov automorphism.

Theorem 2.1. Let $\mu: \mathscr{C} \rightarrow \mathbb{R}^{+}$be a map satisfying conditions (i)-(v) above. Then we have

$$
\mu(f)=\frac{1}{2 \pi} \int_{0}^{2 \pi} f d \theta
$$

The proof of this fact will be divided onto a few lemmas. We use the notation $d \lambda=\frac{1}{2 \pi} d \theta$.

Lemma 2.2. Given $\varepsilon>0$ there is $\delta>0$ such that if $f \in \mathscr{C}, f \leqq \mathbb{1}$, and if

$$
\int_{0}^{2 \pi}\left(\mathbb{1}-f_{n}\right) d \lambda \leqq \delta
$$

then $\mu(f)>1-\varepsilon$.

Proof. Assume to the contrary that there are $f_{n} \in \mathscr{C}, f_{n} \leqq \mathbb{1}$, such that

$$
\int(\mathbb{1}-f) d \lambda \leqq 10^{-n}
$$

and $\mu\left(f_{n}\right) \leqq 1-\varepsilon, n \in \mathbb{N}$. Let

$$
g_{n}=\inf _{k \geqq n} f_{k} \in \mathscr{C}
$$

We have

$$
\int\left(\mathbb{1}-g_{n}\right) d \lambda \leqq \sum_{k \geqq n} \int\left(\mathbb{1}-f_{k}\right) d \lambda \leqq 2 \cdot 10^{-n},
$$

and $g_{n} \leqq f_{n}, g_{n} \nearrow g$ with $g$ equal to $\mathbb{1}$ almost everywhere. Hence $\mu\left(f_{n}\right) \geqq \mu\left(g_{n}\right)$, $\mu\left(g_{n}\right) \nearrow \mu(g)$. Thus $\mu(g)=\mu(\mathbb{1})=1$, contradicting $\mu\left(f_{n}\right) \leqq 1-\varepsilon$.

Lemma 2.3. Given $\varepsilon>0$ there is $\delta>0$ such that the following holds. If $f \in \mathscr{C}$ satisfies $T_{q} f=p \mathbb{1}$ almost everywhere $(p, q \in \mathbb{N})$, and $g \in \mathscr{C}$ satisfies $g \leqq f$ and

$$
\int g d \lambda \geqq(1-\delta) \int f d \lambda,
$$

then we have

$$
\mu(g) \geqq(1-\varepsilon) \int f d \lambda
$$

( $\delta$ depends only on $\varepsilon$ ). 
Proof. Since $d \lambda$ is the Haar measure $\int T_{q} f d \lambda=q \int f d \lambda$, and by (iv) $\mu\left(T_{n} f\right)=n \mu(f)$, we may replace $f$ and $g$ by $T_{q} f$ and $T_{q} g$ respectively. So what must be proved is that there is $\delta>0$ depending only on $\varepsilon$ such that whenever $g \leqq p \mathbb{1}$ for some $p \in \mathbb{N}$ and

then we have

$$
\int g d \lambda \geqq(1-\delta) p,
$$

$$
\mu(g) \geqq(1-\varepsilon) p \text {. }
$$

Using (iv) this follows from Lemma 2.2 and the fact that there is $g_{1} \in \mathscr{C}$ such that $g_{1} \leqq \mathbb{1}$ and $T_{p} g_{1}=g$.

Lemma 2.4. Let $g \in \mathscr{C}$ be upper semicontinuous. Then we have

$$
\mu(g)=\int g d \lambda .
$$

Proof. Let $g_{n}=g \wedge(n \mathbb{1})$. Since $g_{n}>g$ it will be sufficient to prove the lemma for the $g_{n}$ 's, i.e. we may assume $g$ is bounded, say $g \leqq n \mathbb{1}$. Let $\varepsilon>0$. Let $X_{k}=g^{-1}([k, \infty))$. Then $X_{k}$ is compact. It is easily seen that there are $q_{k} \in \mathbb{N}$ and an open set $Y_{k} \supset X_{k}$ such that the boundary of $Y_{k}$ is contained in the set

$$
\left\{e^{2 \pi i s / q_{k}}: 1 \leqq s \leqq q_{k}, s \in \mathbb{N}\right\},
$$

and $\lambda\left(Y_{k}\right)(1-\delta) \leqq \lambda\left(X_{k}\right), \delta$ being as in Lemma 2.3. Let $\chi_{k}$ be the characteristic function $\chi_{Y_{k}}$ of $Y_{k}$, and let

$$
f=\sum_{1 \leqq k \leqq n} \chi_{k}, \quad q=q_{1}, q_{2}, \ldots, q_{n} .
$$

Then $f \geqq g, T_{q} f=p \mathbb{1}$ for some $p \in \mathbb{N}$, and

so that by Lemma 2.3 .

$$
(1-\delta) \int f d \lambda \leqq \int g d \lambda
$$

$$
\mu(g) \geqq(1-\varepsilon) \int f d \lambda \geqq(1-\varepsilon) \int g d \lambda .
$$

On the other hand

$$
\mu(g) \leqq \mu(f)=\frac{1}{q} \mu\left(T_{q} f\right)=\frac{p}{q}=\int f d \lambda \leqq \frac{1}{1-\delta} \int g d \lambda .
$$

Since $\varepsilon>0$ is arbitrary and we may choose $\delta<\varepsilon$ it follows that

$$
\mu(g)=\int g d \lambda \text {. }
$$

Proof of Theorem. By general measure theory there is a sequence $\left(f_{n}\right)$ in $\mathscr{C}$, $f_{1} \leqq f_{2} \leqq \cdots$ such that the $f_{n}$ 's are upper semicontinuous, and if $g=\lim _{n} f_{n}$ then $g=f$ almost everywhere. Thus we have

$$
\int f d \lambda=\int g d \lambda=\lim _{n} \int f_{n} d \lambda=\lim _{n} \mu\left(f_{n}\right)=\mu(g)=\mu(f) .
$$

\section{Some General Entropy Results}

We collect in this section entropy results which do not involve quasifree states, and which are more or less direct consequences of the theory developed in [3]. To fix our notation we recall the definitions in [3]. 
Let $\mathfrak{U}$ be a unital $C^{*}$-algebra, $C_{1}, \ldots, C_{k}$ finite dimensional $C^{*}$-algebras, and $\gamma_{j}: C_{j} \rightarrow \mathfrak{A}$ a unital completely positive map, $j=1, \ldots, k$. Let $\varphi$ be a state on $\mathfrak{U}$ and $P: \mathfrak{U} \rightarrow B$ a unital positive map of $\mathfrak{A}$ into a finite dimensional abelian $C^{*}$-algebra $B$ such that there is a state $\mu$ on $B$ for which $\mu \circ P=\varphi$. If $p_{1}, \ldots, p_{r}$ are the minimal projections in $B$ then there are states $\varphi_{i}, i=1, \ldots, r$, of $\mathfrak{A}$ such that

Since $\mu \circ P=\varphi$,

$$
P(x)=\sum_{i=1}^{r} \varphi_{i}(x) p_{i}, \quad x \in \mathfrak{U} .
$$

$$
\varphi=\sum_{i=1}^{r} \mu\left(p_{i}\right) \varphi_{i}
$$

is $\varphi$ written as a convex combination of the $\varphi_{i}$. In the notation of [3]

$$
\varepsilon_{\mu}(P)=\sum \mu\left(p_{i}\right) S\left(\varphi \mid \varphi_{i}\right)
$$

where $S\left(\varphi \mid \varphi_{i}\right)$ is the relative entropy of the two states $\varphi$ and $\varphi_{i}$, see $[3,6,9]$. The entropy defect $s_{\mu}(P)$ is given by

$$
s_{\mu}(P)=S(\mu)-\varepsilon_{\mu}(P)
$$

where $S(\mu)=-\sum_{i=1}^{r} \mu\left(p_{i}\right) \log \mu\left(p_{i}\right)$ is the entropy of $\mu$.

Let $B_{j}, j=1, \ldots, k$, be a $C^{*}$-subalgebra of $B$ and $E_{j}: B \rightarrow B_{j}$ a $\mu$-invariant conditional expectation. Then the quadruple $\left(B, E_{j}, P, \mu\right)$ is called an abelian model for $\left(\mathfrak{A}, \varphi, \gamma_{1}, \ldots, \gamma_{k}\right)$, and its entropy is defined to be

$$
S\left(\mu \mid \bigvee_{j=1}^{k} B_{j}\right)-\sum_{j=1}^{k} s_{\mu}\left(\rho_{j}\right)
$$

where $\rho_{j}=E_{j} \circ P \circ \gamma_{j}: C_{j} \rightarrow B_{j}$. The sup of the entropies of all such abelian models is denoted by

$$
H_{\varphi}\left(\gamma_{1}, \ldots, \gamma_{k}\right)
$$

If $\alpha$ is a $\varphi$-invariant automorphism of $\mathfrak{A}$ let $\gamma: C \rightarrow \mathfrak{A}$ be a unital completely positive map of a finite dimensional $C^{*}$-algebra $C$, and denote by

$$
h_{\varphi, \alpha}(\gamma)=\lim _{k \rightarrow \infty} \frac{1}{k} H_{\varphi}\left(\gamma, \alpha \circ \gamma, \ldots, \alpha^{k-1} \circ \gamma\right) \text {. }
$$

The entropy of $\alpha$ with respect to $\varphi$ is

$$
h_{\varphi}(\alpha)=\sup _{\gamma} h_{\varphi, \alpha}(\gamma) \text {. }
$$

In the above discussion we have implicitly assumed that the state $\mu$ is faithful. We shall use this assumption explicitly in the proof of our next lemma. But the reader should have no great difficulties in extending the proof to the possible situation of a nonfaithful $\mu$.

Lemma 3.1. Let $\varphi$ be a pure state on the unital $C^{*}$-algebra $\mathfrak{A}$, and suppose $\alpha$ is a $\varphi$-invariant automorphism of $\mathfrak{U}$. Then $h_{\varphi}(\alpha)=0$.

Proof. Let notation be as above with $\left(B, E_{j}, P, \mu\right)$ as the given abelian model. Since $\mu$ is faithful and $P$ is given by (1) it follows from (2) that $\varphi_{i}=\varphi$ for all $i$ since $\varphi$ is 
pure. Thus $P(x)=\varphi(x) 1$ and therefore $\rho_{j}(y)=\varphi \circ \gamma_{j}(y) 1, j=1, \ldots, k$. Consequently $\varepsilon_{\mu}\left(\rho_{j}\right)=0$, and so $s_{\mu}\left(\rho_{j}\right)=S\left(\mu \mid B_{j}\right)$. Thus the entropy for the abelian model is

$$
S\left(\mu \mid \underset{j=1}{\stackrel{r}{\vee}} B_{j}\right)-\sum_{j=1}^{r} S\left(\mu \mid B_{j}\right) \leqq 0,
$$

whence

$$
H_{\varphi}\left(\gamma_{1}, \ldots, \gamma_{k}\right)=0
$$

Since this holds for all choices of $\gamma^{\prime} s, h_{\varphi}(\alpha)=0$.

Lemma 3.2. Let $\mathfrak{A}$ be a $C^{*}$-algebra, $\varphi$ a state, and $\alpha$ a $\varphi$-invariant automorphism. Suppose $\mathscr{B}$ is a $C^{*}$-subalgebra of $\mathfrak{U}$ such that there is an expectation $E: \mathfrak{A} \rightarrow \mathscr{B}$ satisfying $\varphi \circ E=\varphi$, and $\alpha E=E \alpha$. Then $\alpha \mid \mathscr{B}$ is an automorphism of $\mathscr{B}$ and

$$
h_{\varphi}(\alpha \mid \mathscr{B}) \leqq h_{\varphi}(\alpha) \text {. }
$$

Proof. If $C$ is a finite dimensional $C^{*}$-algebra and $\gamma: C \rightarrow \mathfrak{A}$ is completely positive then $E \alpha^{n} \gamma=\alpha^{n} E \gamma$, so by [3, Proposition III.6(b)] and the assumption $\varphi E=\varphi$,

$$
H_{\varphi}\left(E \gamma, \alpha E \gamma, \ldots, \alpha^{k-1} E \gamma\right)=H_{\varphi}\left(E \gamma, E \alpha \gamma, \ldots, E \alpha^{k-1} \gamma\right) \leqq H_{\varphi}\left(\gamma, \alpha \gamma, \ldots, \alpha^{k-1} \gamma\right) .
$$

Thus we have

$$
h_{\varphi ; \alpha \mid \% \delta}(E \gamma) \leqq h_{\varphi, \alpha}(\gamma),
$$

proving the lemma, as it is obvious that $\alpha(\mathscr{B})=\mathscr{B}$.

Lemma 3.3. Let $\mathfrak{U}$ be a $C^{*}$-algebra, $\varphi$ a state, and $\alpha$ a $\varphi$-invariant automorphism of $\mathfrak{U}$. For each $j \in \mathbb{N}$ let $\mathfrak{U}_{j}$ be a $C^{*}$-subalgebra of $\mathfrak{U}$ and $E_{j}: \mathfrak{U} \rightarrow \mathfrak{U}_{j}$ an expectation such that $\alpha E_{j}=E_{j} \alpha$. Suppose $\mathfrak{A}_{1} \subset \mathfrak{U}_{2} \subset \cdots$ is increasing such that

(i) $\mathfrak{A}=\left(\bigcup_{i=1}^{\infty} \mathfrak{U}_{j}\right)^{-}$, norm closure.

(ii) $E_{j+1} E_{j}=E_{j} E_{j+1}=E_{j}, j \in N$.

(iii) $E_{j} \rightarrow$ id pointwise-norm.

Then $\alpha \mid \mathfrak{U}_{j}$ is an automorphism of $\mathfrak{A}_{j}$ for $j \in N$ and

$$
h_{\varphi}(\alpha) \leqq \underline{\lim } h_{\varphi}\left(\alpha \mid \mathfrak{A}_{j}\right) .
$$

If moreover $\varphi \circ E_{j}=\varphi$ for all $j$ then

$$
h_{\varphi}(\alpha)=\lim h_{\varphi}\left(\alpha \mid \mathfrak{U}_{j}\right)
$$

Proof. Since $\alpha E_{j}=E_{j} \alpha, \alpha \mid \mathfrak{U}_{j} \in$ Aut $\left(\mathfrak{U}_{j}\right)$ for all $j$. Let $C$ be a finite dimensional $C^{*}$-algebra, $d=\operatorname{dim} C$, and suppose $\gamma: C \rightarrow \mathfrak{A}$ is a completely positive map. Since $E_{j} \rightarrow$ id, the identity map on $\mathfrak{U}$, positive in norm and $C$ is finite dimensional, $E_{j} \gamma \rightarrow \gamma$ in norm. Furthermore we have

$$
\varphi E_{j}=(\varphi \alpha) E_{j}=\left(\varphi E_{j}\right) \alpha
$$

so that $\alpha$ is $\varphi E_{j}$-invariant for all $j$. Let

$$
\varepsilon_{j}=\left\|E_{j} \gamma-\gamma\right\| \text {. }
$$

Then $\varepsilon_{j} \rightarrow 0$ as $j \rightarrow \infty$. By [3, Proposition IV.3] we have

$$
\left|H_{\varphi}\left(E_{j} \gamma, \alpha E_{j} \gamma, \ldots, \alpha^{k-1} E_{j} \gamma\right)-H_{\varphi}\left(\gamma, \alpha \gamma, \ldots, \alpha^{k-1} \gamma\right)\right| \leqq 6 k \varepsilon_{j}\left(\frac{1}{2}+\log \left(1+d \varepsilon_{j}^{-1}\right)\right),
$$


whence, letting $k \rightarrow \infty$,

$$
\left|h_{\varphi, \alpha \mid \mathscr{U}_{j}}\left(E_{j} \gamma\right)-h_{\varphi, \alpha}(\gamma)\right| \leqq 6 \varepsilon_{j}\left(\frac{1}{2}+\log \left(1+d \varepsilon_{j}^{-1}\right)\right) .
$$

Let $\delta>0$ and choose $C$ and $\gamma$ such that if $h_{\varphi}(\alpha)<\infty$

$$
\left|h_{\varphi}(\alpha)-h_{\varphi, \alpha}(\gamma)\right|<\delta
$$

and if $h_{\varphi}(\alpha)=\infty, h_{\varphi, \alpha}(\gamma) \geqq n$. By (3) we have for this choice of $\gamma$

$$
\begin{aligned}
\left|h_{\varphi, \alpha \mid \mathscr{U}_{j}}\left(E_{j} \gamma\right)-h_{\varphi}(\alpha)\right| & \leqq\left|h_{\varphi, \alpha \mid \mathscr{U}_{j}}\left(E_{j} \gamma\right)-h_{\varphi, \alpha}(\gamma)\right|+\left|h_{\varphi, \alpha}(\gamma)-h_{\varphi}(\alpha)\right| \\
& <6 \varepsilon_{j}\left(\frac{1}{2}+\log \left(1+d \varepsilon_{j}^{-1}\right)\right)+\delta,
\end{aligned}
$$

when $h_{\varphi}(\alpha)<\alpha$, whence

$$
h_{\varphi}\left(\alpha \mid \mathfrak{A}_{j}\right) \geqq h_{\varphi, \alpha \mid \mathfrak{A}_{j}}\left(E_{j} \gamma\right)>h_{\varphi}(\alpha)-6 \varepsilon_{j}\left(\frac{1}{2}+\log \left(1+d \varepsilon_{j}^{-1}\right)\right)-\delta .
$$

If $h_{\varphi}(\alpha)=\infty$ we similarly obtain $h_{\varphi}\left(\alpha \mid \mathfrak{U}_{j}\right) \geqq n-6 \varepsilon_{j}\left(\frac{1}{2}+\log \left(1+d \varepsilon_{j}^{-1}\right)\right)$. Since $6 \varepsilon_{j}\left(\frac{1}{2}+\log \left(1+d \varepsilon_{j}^{-1}\right)\right) \rightarrow 0$ as $j \rightarrow \infty$, we have

$$
\underline{\lim } h_{\varphi}\left(\alpha \mid \mathfrak{U}_{j}\right) \geqq h_{\varphi}(\alpha)-\delta .
$$

Since $\delta$ is arbitrary the first conclusion of the lemma follows.

If $\varphi E_{j}=\varphi$ for all $j$ then the converse inequality $h_{\varphi}(\alpha) \geqq \varlimsup \lim h_{\varphi}\left(\alpha \mid \mathfrak{U}_{j}\right)$ is a consequence of Lemma 3.2.

One of the challenging open problems concerning noncommutative entropy is whether it is additive on tensor product, i.e. is

$$
h_{\varphi \otimes \psi}(\alpha \otimes \beta)=h_{\varphi}(\alpha)+h_{\psi}(\beta) ?
$$

We next show the easy half of this problem.

Lemma 3.4. Let $\mathfrak{U}^{\prime}$ and $\mathfrak{U}^{\prime \prime}$ be two $C^{*}$-algebras with states $\varphi^{\prime}$ and $\varphi^{\prime \prime}$ respectively. Let $\alpha^{\prime}$ and $\alpha^{\prime \prime}$ be $\varphi^{\prime}$ and $\varphi^{\prime \prime}$-invariant automorphisms of $\mathfrak{U}^{\prime}$ and $\mathfrak{U}^{\prime \prime}$. Then

$$
h_{\varphi^{\prime} \otimes \varphi^{\prime \prime}}\left(\alpha^{\prime} \otimes \alpha^{\prime \prime}\right) \geqq h_{\varphi^{\prime}}\left(\alpha^{\prime}\right)+h_{\varphi^{\prime \prime}}\left(\alpha^{\prime \prime}\right) .
$$

Proof. Let $\left(\mathfrak{U}^{\prime}, \varphi^{\prime}, \gamma^{\prime}\right)$ be given with an abelian model $\left(B^{\prime}, E_{j}^{\prime}, P^{\prime}, \mu^{\prime}\right)$ and subalgebras $B_{j}^{\prime}$ with $E_{j}^{\prime}$ the $\mu^{\prime}$-invariant expectation of $B^{\prime}$ onto $B_{j}^{\prime}$. Assume we have a similar setup for $\left(\mathfrak{U}^{\prime \prime}, \varphi^{\prime \prime}, \gamma^{\prime \prime}\right)$. Since relative entropy and entropy of states are additive on tensor products we have additivity of $\varepsilon_{\mu}, S(\mu), s_{\mu}$. We may assume $B^{\prime}=\bigvee_{i=1}^{k} B_{i}^{\prime}$, $B^{\prime \prime}=\bigvee_{i=1}^{k} B_{i}^{\prime \prime}$, and so $B^{\prime} \otimes B^{\prime \prime}=\bigvee_{i=1}^{k} B_{i}^{\prime} \otimes B_{i}^{\prime \prime}$. Thus

$$
S\left(\mu^{\prime} \otimes \mu^{\prime \prime} \mid \bigvee_{i=1}^{k} B_{i}^{\prime} \otimes B_{i}^{\prime \prime}\right)=S\left(\mu^{\prime}\right)+S\left(\mu^{\prime \prime}\right)
$$

It follows easily that the entropy of the tensor product of the abelian model for $\left(\mathfrak{U}^{\prime} \otimes \mathfrak{U}^{\prime \prime}, \varphi^{\prime} \otimes \varphi^{\prime \prime},\left(\alpha^{\prime}\right)^{j_{\circ}} \gamma^{\prime} \otimes\left(\alpha^{\prime \prime}\right)^{j_{\circ}} \gamma^{\prime \prime}, j=0, \ldots, k-1\right)$ is the sum of the entropies of the two abelian models for $\mathfrak{U}^{\prime}$ and $\mathfrak{U}^{\prime \prime}$ respectively. Taking sup over all tensor product abelian models as above we get

$$
H_{\varphi^{\prime}}\left(\gamma^{\prime}, \alpha^{\prime} \gamma^{\prime}, \ldots,\left(\alpha^{\prime}\right)^{k-1} \gamma^{\prime}\right)+H_{\varphi^{\prime \prime}}\left(\gamma^{\prime \prime}, \alpha^{\prime \prime} \gamma^{\prime \prime}, \ldots,\left(\alpha^{\prime \prime}\right)^{k-1} \gamma^{\prime \prime}\right) .
$$

However, to get

$$
H_{\varphi^{\prime} \otimes \varphi^{\prime \prime}}\left(\gamma^{\prime} \otimes \gamma^{\prime},\left(\alpha^{\prime} \otimes \alpha^{\prime \prime}\right)\left(\gamma^{\prime} \otimes \gamma^{\prime \prime}\right), \ldots,\left(\alpha^{\prime} \otimes \alpha^{\prime \prime}\right)^{k-1}\left(\gamma^{\prime} \otimes \gamma^{\prime \prime}\right)\right)
$$


we take the sup over a larger family of abelian models. Thus the expression in (5) is greater than that in (4). Similarly, taking the sup of all possible $\gamma$ 's the conclusion of the lemma follows.

\section{Bogoliubov Automorphisms and Quasifree States}

Let $H$ be a complex Hilbert space. The CAR-algebra $\mathscr{A}(H)$ over $H$ is a $C^{*}$-algebra with the property that there is a linear map $f \rightarrow a(f)$ of $H$ into $\mathscr{A}(H)$ whose range generates $\mathscr{A}(H)$ as a $C^{*}$-algebra and satisfying the canonical anticommutation relations

$$
\begin{aligned}
a(f) a(g)^{*}+a(g)^{*} a(f) & =(f, g) 1, \quad f, g \in H, \\
a(f) a(g)+a(g) a(f) & =0,
\end{aligned}
$$

where $(\cdot, \cdot)$ is the inner product on $H$, and 1 is the unit of $\mathscr{A}(H)$. If $0 \leqq A \leqq 1$ is an operator on $H$ then the quasifree state $\omega_{A}$ on $\mathfrak{A}(H)$ is defined by its values on products of the form $a\left(f_{n}\right)^{*} \cdots a\left(f_{1}\right)^{*} a\left(g_{1}\right) \cdots a\left(g_{m}\right)$ given by

$$
\omega_{A}\left(a\left(f_{n}\right) * \cdots a\left(f_{1}\right) * a\left(g_{1}\right) \cdots a\left(g_{m}\right)\right)=\delta_{n m} \operatorname{det}\left(\left(A g_{i}, f_{j}\right)\right) \text {. }
$$

If $U$ is a unitary operator on $H$ then $U$ defines an automorphism $\alpha_{U}$ of $\mathscr{A}(H)$, called a Bogoliubov automorphism (or quasifree or one-particle automorphism) determined by

$$
\alpha_{U}(a(f))=a(U f) .
$$

If $U$ and $A$ commute it is an easy consequence of the above definition of $\omega_{A}$ that $\alpha_{U}$ is $\omega_{A}$-invariant. More generally if $T$ is a contraction on $H$ commuting with $A$ then there is a unique unital completely positive map $\alpha_{T}: \mathscr{A}(H) \rightarrow \mathscr{A}(H)$ such that $\alpha_{T}(a(f))=a(T f)$, and $\omega_{A} \alpha_{T}=\omega_{A}$, see [5]. If $P$ is a projection commuting with $A$ then $\alpha_{P}$ is an expectation of $\mathscr{A}(H)$ onto $\mathscr{A}(P H)$.

We shall mostly be concerned with the case when $A$ has pure point spectrum, say $\left(f_{n}\right)_{n \in \mathbb{N}}$ is an orthonormal basis for $H$ such that $A f_{n}=\lambda_{n} f_{n}, n \in \mathbb{N}$. Define recursively $V_{0}=1, V_{n}=\prod_{i=1}^{n}\left(1-2 a\left(f_{i}\right)^{*} a\left(f_{i}\right)\right), e_{11}^{(n)}=a\left(f_{n}\right) a\left(f_{n}\right)^{*}, e_{12}^{(n)}=a\left(f_{n}\right) V_{n-1}$, $e_{21}^{(n)}=V_{n-1} a\left(f_{n}\right)^{*}, e_{22}^{(n)}=a\left(f_{n}\right)^{*} a\left(f_{n}\right)$. Then the $\left(e_{i j}^{(n)}: i=1,2\right)$ form a complete set of $2 \times 2$ matrix units generating a factor $M_{2}(\mathbb{C})_{n}$ of type $I_{2}$, and for distinct $n, m e_{i j}^{(m)}$ and $e_{k l}^{(n)}$ commute. It follows in particular that $\mathscr{A}(H) \cong \bigotimes_{n=1}^{\infty} M_{2}(\mathbb{C})_{n}$ and that $\omega_{A}$ is a product state, $\omega_{A}=\bigotimes_{n=1}^{\infty} \omega_{\lambda_{n}}$ with respect to this tensor product factorization, where $\omega_{\lambda}$ is the state on $M_{2}(\mathbb{C})$ given by

$$
\omega_{\lambda}\left(\left(\begin{array}{ll}
a & b \\
c & d
\end{array}\right)\right)=(1-\lambda) a+\lambda d .
$$

The Bogoliubov automorphism $\alpha_{-1}$ is $\omega_{A}$-invariant for all quasifree states $\omega_{A}$. Its fixed point algebra is denoted by $\mathscr{A}(H)_{e}$ and is the even CAR-algebra. It is generated by even products of $a(f)$ 's and $a(g)^{*}$ 's. $\mathscr{A}(H)$ is the direct sum of $\mathscr{A}(H)_{e}$ and the spectral subspace of -1 for $\alpha_{-1}$. If $H=H_{1} \oplus H_{2}$ then

$$
\mathscr{A}\left(H_{2}\right)_{e} \subset \mathscr{A}\left(H_{1}\right)^{\prime} \cap \mathscr{A}(H) \text {, }
$$


because operators $a(f)^{\#}, f \in H_{1}$, and $a(g)^{\#}, g \in H_{2}$, anticommute, where $a^{\#}$ denotes $a^{*}$ or $a$. Thus even products of the $a(g)^{\# ' s ~ w i l l ~ c o m m u t e ~ w i t h ~} a(f)^{\#}$. Thus for each finite even dimensional subspace $K_{n}$ of $H_{1}$ the $C^{*}$-algebra generated by $\mathscr{A}\left(K_{n}\right)$ and $\mathscr{A}\left(\mathrm{H}_{2}\right)_{e}$ is isomorphic to $\mathscr{A}\left(K_{n}\right) \otimes \mathscr{A}\left(H_{2}\right)_{e}$. Since $\mathscr{A}\left(H_{1}\right)$ is the norm closure of the union of such $\mathscr{A}\left(K_{n}\right)$ 's it follows from the uniqueness of the tensor product norm that the $C^{*}$-algebra generated by $\mathscr{A}\left(H_{1}\right)$ and $\mathscr{A}\left(H_{2}\right)_{e}$ is isomorphic to $\mathscr{A}\left(H_{1}\right) \otimes \mathscr{A}\left(H_{2}\right)_{e}$.

Let $A=A_{1} \oplus A_{2}, A_{i} \in B\left(H_{i}\right), 0 \leqq A_{i} \leqq 1$. Then

$$
\omega_{A}\left|\mathscr{A}\left(H_{1}\right) \otimes \mathscr{A}\left(H_{2}\right)_{e}=\omega_{A_{1}}\right| \mathscr{A}\left(H_{1}\right) \otimes \omega_{A_{2}} \mid \mathscr{A}\left(H_{2}\right)_{e} .
$$

Indeed, in (1) let $m=n$ and use the anticommutation relations to rearrange the factors in the defining equation (1) so that $f_{1}, \ldots, f_{k} \in H_{1}, f_{k+1}, \ldots, f_{n} \in H_{2}$, $g_{1}, \ldots, g_{l} \in H_{1}, g_{l+1}, \ldots, g_{n} \in H_{2}$. Since $\left(A g_{i}, f_{j}\right)=0$ if one of $g_{i}$ and $f_{j}$ is in $H_{1}$ and the other in $\mathrm{H}_{2}$, the matrix $\left(\left(A g_{i}, f_{j}\right)\right)$ is a block matrix

$$
\left(\begin{array}{cc}
\left(\left(A_{1} g_{i}, f_{j}\right)\right) & 0 \\
0 & \left(\left(A_{2} g_{i}, f_{j}\right)\right)
\end{array}\right),
$$

where $\left(\left(A_{1} g_{i}, f_{j}\right)\right)$ is a $k \times l$ matrix and $\left(\left(A_{2} g_{i}, f_{j}\right)\right)$ and $(n-k) \times(n-l)$ matrix. The determinant of this matrix is zero unless $k=l$, a fact easily verified by induction. If $k=l$ the determinant equals the product

$$
\omega_{A_{1}}\left(a\left(f_{k}\right) * \ldots a\left(f_{1}\right) * a\left(g_{1}\right) \cdots a\left(g_{k}\right)\right) \omega_{A_{2}}\left(a\left(f_{n}\right) * \cdots a\left(f_{k+1}\right) * a\left(g_{k+1}\right) \cdots a\left(g_{n}\right)\right),
$$

from which (2) follows.

Suppose next $U_{i}$ is a unitary operator on $H_{i}, i=1,2$. If $f \in H_{1}, U_{1} \oplus U_{2} f=U_{1} f$ with obvious identification of $f$ and $f \oplus 0$. Thus $\alpha_{U_{1} \oplus U_{2}}(a(f))=\alpha_{U_{1}}(a(f))$ and similarly for $g \in H_{2}$. If $f_{1}, \ldots, f_{n} \in H_{1}, g_{1}, \ldots, g_{m} \in H_{2}$ we have, with \# as before,

$$
\begin{aligned}
\alpha_{U_{1} \oplus U_{2}}\left(\prod_{i=1}^{n} a\left(f_{i}\right)^{\#} \prod_{j=1}^{m} a\left(g_{j}\right)^{\#}\right) & =\prod_{i} a\left(U_{1} f_{i}\right)^{\#} \prod_{j} a\left(U_{2} g_{j}\right)^{\#} \\
& =\alpha_{U_{1}}\left(\prod_{i} a\left(f_{i}\right)^{\#}\right) \alpha_{U_{2}}\left(\prod_{j} a\left(g_{j}\right)^{\#}\right) .
\end{aligned}
$$

It follows that

$$
\alpha_{U_{1} \oplus U_{2}}\left|\mathscr{A}\left(H_{1}\right) \otimes \mathscr{A}\left(H_{2}\right)_{e}=\alpha_{U_{1}}\right| \mathscr{A}\left(H_{1}\right) \otimes \alpha_{U_{2}} \mid \mathscr{A}\left(H_{2}\right)_{e}
$$

Lemma 4.1. Let $H=H_{1} \oplus H_{2}, 0 \leqq A_{i} \leqq 1$ be an operator on $H_{i}$, and $U_{i}$ be a unitary operator on $H_{i}, i=1,2$. Suppose $A_{i} U_{i}=U_{i} A_{i}, i=1,2$. Then we have

$$
h_{\omega_{A_{1} \oplus A_{2}}}\left(\alpha_{U_{1} \oplus U_{2}}\right) \geqq h_{\omega_{A_{1}}}\left(\alpha_{U_{1}}\right) \text {. }
$$

Proof. Let $E: \mathscr{A}\left(H_{1} \oplus H_{2}\right) \rightarrow \mathscr{A}\left(H_{1}\right) \otimes \mathscr{A}\left(H_{2}\right)_{e}$ be the expectation $E=\frac{1}{2}\left(\mathrm{id}+\alpha_{1 \oplus-1}\right)$, 1 denoting the identity on both $H_{1}$ and $H_{2}$. Since $1 \oplus-1$ commutes with $A_{1} \oplus A_{2}, \alpha_{1 \oplus-1}$ is $\omega_{A_{1} \oplus A_{2}}$-invariant, as is $E$. Thus by Lemmas 3.2 and 3.4 we have

$$
\begin{aligned}
h_{\omega_{A_{1} \oplus A_{2}}}\left(\alpha_{U_{1} \oplus U_{2}}\right) \geqq h_{\omega_{A_{1} \oplus A_{2}}}\left(\alpha_{U_{1} \oplus U_{2}} \mid \mathscr{A}\left(H_{1}\right) \otimes \mathscr{A}\left(H_{2}\right)_{e}\right) \\
=h_{\omega_{A_{1}} \otimes \omega_{A_{2}}}\left(\alpha_{U_{1}} \otimes \alpha_{U_{2}} \mid \mathscr{A}\left(H_{1}\right) \otimes \mathscr{A}\left(H_{2}\right)_{e}\right) \\
\geqq h_{\omega_{A_{1}}}\left(\alpha_{U_{1}} \mid \mathscr{A}\left(H_{1}\right)\right)+h_{\omega_{A_{2}}}\left(\alpha_{U_{2}} \mid \mathscr{A}\left(H_{2}\right)_{e}\right) \\
\geqq h_{\omega_{A_{1}}}\left(\alpha_{U_{1}} \mid \mathscr{A}\left(H_{1}\right)\right) . \quad \square
\end{aligned}
$$


Remark 4.2. We remark for later use that it follows from the discussion preceding the lemma that if $H=\bigoplus_{i \in J} H_{i}, J$ finite, then $\mathscr{A}(H)_{e} \supset \bigotimes_{i \in J} \mathscr{A}\left(H_{i}\right)_{e}$, and if $U=\bigoplus_{i \in J} U_{i}$ is unitary and $A=\bigoplus_{i \in J} A_{i}$ satisfies $0 \leqq A \leqq 1$, then

and

$$
\omega_{A} \mid \bigotimes_{i \in J} \mathscr{A}\left(H_{i}\right)_{e}=\bigotimes_{i \in J}\left(\omega_{A_{i}} \mid \mathscr{A}\left(H_{i}\right)_{e}\right)
$$

$$
\alpha_{U} \mid \bigotimes_{i \in J} \mathscr{A}\left(H_{i}\right)_{e}=\bigotimes_{i \in J}\left(\alpha_{U_{i}} \mid \mathscr{A}\left(H_{i}\right)_{e}\right)
$$

Furthermore, if $G$ is the group of unitaries $U=\bigoplus_{i \in J} U_{i}$ with $U_{i}= \pm 1$, then $E=2^{- \text {card } J} \sum_{U \in G} \operatorname{Ad} U$ is an $\omega_{A}$-invariant expectation of $\mathscr{A}(H)$ onto $\bigotimes_{i \in J} \mathscr{A}\left(H_{i}\right)_{e}$.

Each unitary operator $U$ is a direct sum $U=U_{a} \oplus U_{s}$ with $U_{a}$ acting on a Hilbert space $H_{a}$ and $U_{s}$ on $H_{s} ; U_{a}$ has spectral measure absolutely continuous with respect to Lebesgue measure $d \theta$ on the circle $\mathbf{T}$ while $U_{s}$ has spectral measure singular with respect to $d \theta . U_{a}$ is called the absolutely continuous part of $U$ and $U_{s}$ the singular part. We shall in the sequel use the notation $m(U)$ to denote the multiplicity function of $U_{a}$, i.e. $m(U)=m\left(U_{a}\right)$ in our notation.

Lemma 4.3. Let $U$ and $V$ be unitary operators and $\lambda \in[0,1]$. Then we have, identifying $\lambda$ and $\lambda 1$,

(i) If there is a unitary operator $W$ such that $V=W U W^{-1}$ then $h_{\omega_{\lambda}}\left(\alpha_{U}\right)=h_{\omega_{\lambda}}\left(\alpha_{V}\right)$.

(ii) If $U$ and $V$ have the same singular parts and $m(U) \geqq m(V)$, then $h_{\omega_{\lambda}}\left(\alpha_{U}\right) \geqq h_{\omega_{\lambda}}\left(\alpha_{V}\right)$.

Proof. (i) is obvious, cf. [3, VII.5].

(ii) The assumption on $U$ and $V$ means that up to unitary equivalence we may assume $V$ is the restriction of $U$ to a reducing subspace, so that (ii) follows from Lemma 4.1 .

Lemma 4.4. Let $\left(U_{n}\right)$ be a sequence of unitary operators and $U$ a unitary operator, all with Lebesgue spectrum. Suppose $\left(m\left(U_{n}\right)\right)$ is an increasing sequence with pointwise limit $m(U)$. Then $\left(h_{\omega_{\lambda}}\left(\alpha_{U_{n}}\right)\right)$ is an increasing sequence and

$$
h_{\omega_{\lambda}}\left(\alpha_{U}\right)=\lim _{n \rightarrow \infty} h_{\omega_{\lambda}}\left(\alpha_{U_{n}}\right) \text {. }
$$

Proof. Since the singular part of each unitary $n$ question is zero the assumption on the multiplicity functions implies that we may assume $U$ lives on a Hilbert space $H$ and $U_{n}=\frac{U \mid H_{n}}{\infty}$, where $H_{n} \subset H_{n+1} \subset H$ are reducing subspaces for $U$ for all $n \in \mathbb{N}$, and $H=\bigcup_{n=1} H_{n}$. Thus the lemma follows from Lemmas 3.2 and 3.4 and the fact that the projections onto the $H_{n}$ define expectations on $\mathscr{A}(H)$ satisfying the conditions in the lemmas.

We conclude this section with the computation of $h_{\omega_{A}}\left(\alpha_{U}\right)$ in some simple cases.

Lemma 4.5. For $i \in\{1, \ldots, r\}$ let $H_{i}$ be an infinite dimensional separable Hilbert space with identity $1_{i}$ and let $U_{i}$ be a unitary operator on $H_{i}$ such that for each $i$ there are $p_{i} \in \mathbb{N}$ and a common $q \in \mathbb{N}$ for all $i$, such that $U_{i}^{q}$ is unitarily equivalent to $V^{p_{i}}$, where $V$ is a bilateral shift operator of multiplicity 1 . Let $U=\bigoplus_{i=1}^{r} U_{i}$ and let $A=\bigoplus_{i=1}^{r} c_{i} 1_{i}$ 
with $c_{i} \in[0,1]$. Then we have the formula

$$
h_{\omega_{A}}\left(\alpha_{U}\right)=q^{-1} \sum_{i=1}^{r} p_{i} S\left(\omega_{c_{i}}\right)=q^{-1} \sum_{i=1}^{r} p_{i}\left(\eta\left(c_{i}\right)+\eta\left(1-c_{i}\right)\right) .
$$

Furthermore, the same formula holds for the restrictions of $\omega_{A}$ and $\alpha_{U}$ to $\mathscr{A}(H)_{e}$. Proof. Let $\left(f_{i k}\right)_{k \in \mathbb{Z}}$ be an orthonormal basis for $H_{i}$ such that $V f_{i k}=f_{i(k+1)}$, and let

$$
N=\mathscr{A}\left(\left[f_{11}, \ldots, f_{1 p_{1}}, \ldots, f_{r 1}, \ldots, f_{r p_{r}}\right]\right),
$$

where $\left[f_{1}, \ldots, f_{m}\right]$ denotes the subspace spanned by the vectors $f_{1}, \ldots, f_{m}$. Since $A f_{i k}=c_{i} f_{i k}$ we can write $N$ as a tensor product

$$
N=\bigotimes_{i=1}^{p} M_{2}(\mathbb{C})_{i}
$$

where $p=\sum_{i=1}^{r} p_{i}$, and from our previous discussion

$$
\omega_{A} \mid N=\left(\bigotimes_{i=1}^{p_{1}} \omega_{c_{1}}\right) \otimes \cdots \otimes\left(\bigotimes_{i=1}^{p_{r}} \omega_{c_{r}}\right) .
$$

The subspaces

$$
\left(U^{q}\right)^{k}\left(\left[f_{11}, \ldots, f_{r p_{r}}\right]\right)=\bigoplus_{i=1}^{r} V^{k p_{1}}\left(\left[f_{i 1}, \ldots, f_{i p_{i}}\right]\right)
$$

for $k \in \mathbb{Z}$ are mutually orthogonal since $V$ is a bilateral shift and the spaces $H_{i}$ are left invariant. Furthermore the subspaces span $H=\bigoplus_{i=1}^{r} H_{i}$. Thus the algebras $\left(\alpha_{U^{q}}\right)^{k}(N)$ generate $\mathscr{A}(H)$, and their even parts are pairwise commuting. The diagonals in the algebras $M_{2}\left(\mathbb{C}_{i}\right)$ appearing in the definition of $N$ lie in the centralizer of the corresponding $\omega_{c_{j}}$, hence in the centralizer of $\omega_{A}$. Furthermore, they lie in $\mathscr{A}(H)_{e}$ as follows from the construction of the $M_{2}(\mathbb{C})_{i}$. If $N_{k}$ is the algebra generated by $N, \alpha_{U^{q}}(N), \ldots,\left(\alpha_{U^{q}}\right)^{k-1}(N)$ it thus follows from [3, Corollary VIII.8] that

$$
\begin{aligned}
\frac{1}{k} H_{\omega_{A}}\left(N, \alpha_{U^{q}}(N), \ldots,\left(\alpha_{U^{q}}\right)^{k-1}(N)\right) & =\frac{1}{k} S\left(\omega_{A} \mid N_{k}\right) \\
& =\frac{1}{k} \sum_{i=0}^{k-1} S\left(\omega_{A} \mid\left(\alpha_{U^{q}}\right)^{i}(N)\right) \\
& =S\left(\omega_{A} \mid N\right) \\
& =\sum_{i=1}^{r} p_{i} S\left(\omega_{c_{i}}\right) .
\end{aligned}
$$

Hence we have

$$
h_{\omega_{A}, \alpha_{U} a}(N)=\sum_{i=1}^{r} p_{i} S\left(\omega_{c_{i}}\right) .
$$

To complete the argument let for each $n \in \mathbb{N}$,

$$
M_{n}=\bigvee_{-n}^{n}\left(\alpha_{U^{q}}\right)^{j}(N)
$$


Then $\left(M_{n}\right)$ is an increasing sequence of finite dimensional subalgebras of $\mathscr{A}(H)$ with dense union. Then by [3, Theorem VII.4]

$$
h_{\omega_{A}}\left(\alpha_{U^{q}}\right)=\lim _{n \rightarrow \infty} h_{\omega_{A}, \alpha_{U q}}\left(M_{n}\right) \text {. }
$$

Now the $C^{*}$-algebra generated by $M_{n}, \alpha_{U^{q}}\left(M_{n}\right), \ldots,\left(\alpha_{U^{q}}\right)^{k-1}\left(M_{n}\right)$ equals the one generated by $\left(\alpha_{U q}\right)^{j}(N),-n \leqq j \leqq n+k-1$. Our previous argument with $N$ and the diagonals in the $M_{2}(\mathbb{C})_{i}$ shows that [3, Corollary VIII.8] implies

$$
H_{\omega_{A}}\left(M_{n}, \alpha_{U^{q}}\left(M_{n}\right), \ldots,\left(\alpha_{U^{q}}\right)^{k-1}\left(M_{n}\right)\right)=(2 n+k) S\left(\omega_{A} \mid N\right),
$$

and this holds also for $H_{\omega_{A} \mid \mathscr{A}(H)_{e}}$ and $M_{n} \cap \mathscr{A}(H)_{e}$. We conclude that

$$
h_{\omega_{A}, \alpha_{U} 9}\left(M_{n}\right)=S\left(\omega_{A} \mid N\right) \text {, }
$$

and hence from (3) $h_{\omega_{A}}\left(\alpha_{U^{q}}\right)=S\left(\omega_{A} \mid N\right)$. Thus it follows from [3, VIII.5] that

$$
h_{\omega_{A}}\left(\alpha_{U}\right)=\frac{1}{q} h_{\omega_{A}}\left(\alpha_{U^{q}}\right)=\frac{1}{q} \sum_{i=1}^{r} p_{i} S\left(\omega_{c_{i}}\right) .
$$

Finally, since the diagonals in the $M_{2}(\mathbb{C})_{i}$ lie in $\mathscr{A}(H)_{e}$ we get the inequality

$$
h_{\omega_{A} \mid \mathscr{A}(H)_{e}}\left(\alpha_{U} \mid \mathscr{A}(H)_{e}\right) \geqq \frac{1}{q} \sum_{i=1}^{r} p_{i} S\left(\omega_{c_{i}}\right) .
$$

The opposite inequality follows from (4) and Lemma 3.2.

Lemma 4.6. Let $U$ be a unitary operator on $H$ with Lebesgue spectrum consisting of disjoint arcs $\exp \left(2 \pi i\left[a_{j}, b_{j}\right]\right)$ such that $b_{j}-a_{j}=\frac{p_{j}}{q}, p_{j}, q \in \mathbb{N}$, with $j \in J \subset \mathbb{N}$. Let $H_{j}=L^{2}\left(\exp \left(2 \pi i\left[a_{j}, b_{j}\right]\right)\right)$ considered as a subspace of $L^{2}(\mathbf{T}, d \theta)$, and write $U=\bigoplus_{j \in J} U_{j}$ with $U_{j}=U \mid H_{j}$. Suppose $U_{j}$ has constant finite multiplicity $n_{j}$, and let $0 \leqq A_{j} \leqq 1$ act on $H_{j}$ and commute with $U_{j}$. Writing $U_{j}=V_{j} \oplus \cdots \oplus V_{j}\left(n_{j}\right.$ times) we assume $A_{j}=\bigoplus_{k=1}^{n_{j}} c_{j k} 1_{j}$, where $1_{j}$ is the identity on the space on which $V_{j}$ acts. Let $B_{j}$ denote the diagonal $n_{j} \times n_{j}$ matrix

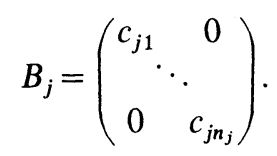

Then $A_{j}=B_{j} \otimes 1_{j}$, and we have the formula

$$
h_{\omega_{A}}\left(\alpha_{U}\right)=\sum_{j \in J}\left(b_{j}-a_{j}\right) \operatorname{Tr}_{n_{j}}\left(\eta\left(B_{j}\right)+\eta\left(1-B_{j}\right)\right),
$$

where $\operatorname{Tr}_{n_{j}}$ is the usual trace on $M_{n_{j}}(\mathbb{C})$. Furthermore, the same formula holds for the restrictions of $\omega_{A}$ and $\alpha_{U}$ to $\mathscr{A}(H)_{e}$.

Proof. We first assume $J$ is finite, say $J=\{1, \ldots, r\}$. We may write

$$
\begin{aligned}
& U=(\underbrace{V_{1} \oplus \cdots \oplus V_{1}}_{n_{1}}) \oplus \cdots \oplus(\underbrace{V_{r} \oplus \cdots \oplus V_{r}}_{n_{r}}) \\
& A=\left(c_{11} 1_{1} \oplus \cdots \oplus c_{1 n_{1}} 1_{1}\right) \oplus \cdots \oplus\left(c_{r 1} 1_{r} \oplus \cdots \oplus c_{r n_{r}} 1_{r}\right) .
\end{aligned}
$$


Now $V_{j}^{q}$ is a bilateral shift of multiplicity $p_{j}$. Thus by Lemma 4.5

$$
\begin{aligned}
h_{\omega_{A}}\left(\alpha_{U}\right) & =\sum_{j=1}^{r} \frac{p_{j}}{q} \sum_{l=1}^{n_{j}} S\left(\omega_{c_{j l}}\right) \\
& =\sum_{j=1}^{r} \frac{p_{j}}{q} \operatorname{Tr}_{n_{j}}\left(\eta\left(B_{j}\right)+\eta\left(1-B_{j}\right)\right) \\
& =\sum_{j=1}^{r}\left(b_{j}-a_{j}\right) \operatorname{Tr}_{n_{j}}\left(\eta\left(B_{j}\right)+\eta\left(1-B_{j}\right)\right) .
\end{aligned}
$$

If $J$ is infinite we may assume $J=\mathbb{N}$. Let $W_{r}=\bigoplus_{j=1}^{r} U_{j}$. Then $W_{r} A=A W_{r}$, so if $Q_{r}$ is the orthogonal projection of $H$ onto $\bigoplus_{j=1}^{r} H_{j}$ then the expectations $E_{r}$ of $\mathscr{A}(H)$ onto $\mathscr{A}\left(\bigoplus_{j=1}^{r} H_{j}\right)$ defined by $Q_{r}$ satisfying the conditions of Lemma 3.3, cf. [5]. Thus by Lemma 3.3 the proof is complete.

\section{The Case of Singular Spectrum}

In this section we study the case when the unitary operator $U$ has a nontrivial singular part $U_{s}$. The main result shows that if $U=U_{a} \oplus U_{s}$ with $U_{a}$ as in Lemma 4.6 then $h_{\omega_{A}}\left(\alpha_{U}\right)=h_{\omega_{A}}\left(\alpha_{U_{a}}\right)$ with $A$ as in that lemma. We first prove an operator theoretic lemma.

Lemma 5.1. Let $U$ be a unitary operator on $H$ with spectral measure singular with respect to Lebesgue measure. Let $P$ be a finite rank orthogonal projection onto a subspace of $H$, and let $\varepsilon>0$ be given. Then there is $k_{0} \in \mathbb{N}$ such that for each integer $k \geqq k_{0}$ there is a finite rank projection $Q_{k}$ with the properties

(i) $\left\|\left(1-Q_{k}\right) U^{s} P\right\|<\varepsilon$, for $0 \leqq s \leqq k$.

(ii) $\operatorname{dim} Q_{k} \leqq \varepsilon k$.

Proof. Since the spectral measure of $U$ is a singular and $P$ has finite rank there is a set $\sigma \subset \mathbf{T}$ such that the following hold for given $\delta>0$ :

(a) $\sigma=\sigma_{1} \cup \sigma_{2} \cup \cdots \cup \sigma_{N}$, where $\sigma_{j}(1 \leqq j \leqq N)$ are $\operatorname{arcs}, \sigma_{j}=\left\{\exp (i \theta): \alpha_{j} \leqq \theta \leqq \beta_{j}\right\}$ such that $0 \leqq \alpha_{1}<\beta_{1} \leqq \alpha_{2}<\beta_{2} \leqq \cdots \leqq \alpha_{N}<\beta_{N} \leqq 2 \pi$.

(b) $N \max \left(\beta_{j}-\alpha_{j}\right)<\delta$.

(c) If $E(\sigma)$ is the spectral projection of $U$ for the set $\sigma$ then $\|(1-E(\sigma)) P\|<\delta$.

Indeed, since the spectral measure of $U$ is singular it is immediate that (a),(c), and the condition $\sum\left(\beta_{j}-\alpha_{j}\right)<\delta$ can be satisfied. To get (b) it suffices to substitute the $\sigma_{j}$ by arcs of almost the same length.

For a number $M>0$ consider the $M N$ arcs $\sigma_{1,1}, \ldots, \sigma_{N M, M}$ obtained by subdividing each $\sigma_{j}$ into $M$ arcs of equal length. Let $E\left(\sigma_{j, M}\right)$ be the corresponding spectral projections of $U$, which are pairwise orthogonal. Let then $\mathscr{X}_{M}$ be the subspace

$$
\mathscr{X}_{M}=\bigoplus_{j=1}^{M N} E\left(\sigma_{j, M}\right) P(H)
$$


We have $\operatorname{dim} \mathscr{X}_{M} \leqq M N \operatorname{dim} P$. If $f \in P(H)$ and $\|f\|=1$, then we have with $\theta_{j} \in \sigma_{j, M}$, that the distance

$$
\begin{aligned}
d\left(U^{s} f, \mathscr{X}_{M}\right)^{2} & \leqq \\
& \leqq 2 \sum_{j=1}^{M N} e^{i \theta_{j} s} E\left(\sigma_{j, M}\right) f-U^{s} f\left\|^{2}\right\| e^{i \theta_{j} s} E\left(\sigma_{j, M}\right) f-U^{s} E\left(\sigma_{j, M}\right) f\left\|^{2}+2\right\| U^{s} E(\sigma) f-U^{s} f \|^{2} \\
& \leqq 2 \sum_{j=1}^{M N}\left\|\left(e^{i \theta_{j} s} 1-U^{s}\right) E\left(\sigma_{j, M}\right)\right\|^{2}\left\|E\left(\sigma_{j, M}\right) f\right\|^{2}+2 \delta^{2} \\
& \leqq 2 \max _{1 \leqq j \leqq M N}\left\|\left(e^{i \theta_{j} s} 1-U^{s}\right) E\left(\sigma_{j, M}\right)\right\|^{2}+2 \delta^{2}
\end{aligned}
$$

On the other hand

$$
\begin{aligned}
\left\|\left(e^{i \theta_{j} s} 1-U^{s}\right) E\left(\sigma_{j, M}\right)\right\| & \leqq \sup _{z \in \sigma_{j, M}}\left|e^{i \theta_{j} s}-z^{s}\right| \\
& \leqq\left(\text { length } \sigma_{j, M}\right) \cdot s \\
& \leqq \frac{s}{M} \max _{1 \leqq j \leqq M N}\left(\beta_{j}-\alpha_{j}\right) \\
& <\frac{s \delta}{M N}
\end{aligned}
$$

This gives

$$
\begin{aligned}
d\left(U^{s} f, \mathscr{X}_{M}\right) & \leqq\left(2\left(\frac{s \delta}{M N}\right)^{2}+2 \delta^{2}\right)^{1 / 2} \\
& \leqq \sqrt{2} \frac{s \delta}{M N}+\sqrt{2} \delta
\end{aligned}
$$

Since this estimate is uniform in $f \in P H,\|f\|=1$, we have actually proved that for $Q$ the orthogonal projection onto $\mathscr{X}_{M}$ we have

and

$$
\left\|(1-Q) U^{s} P\right\|<\sqrt{2}\left(\frac{s}{M N}+1\right) \delta,
$$

$$
\operatorname{dim} Q \leqq M N \operatorname{dim} P \text {. }
$$

Given $k$ let us take $Q_{k}$ to be the orthogonal projection onto $\mathscr{X}_{M}$, and with $\varepsilon$ as in the statement of the lemma, let

$$
M=\left[\frac{\varepsilon k}{N \operatorname{dim} P}\right]
$$

Then $\operatorname{dim} Q_{k} \leqq \varepsilon k$. On the other hand

$$
\max _{1 \leqq s \leqq k} \sqrt{2}\left(\frac{s}{\left[\frac{\varepsilon k}{N \operatorname{dim} P}\right] N}+1\right) \delta=\sqrt{2}\left(\frac{k}{\left[\frac{\varepsilon k}{N \operatorname{dim} P}\right] N}+1\right) \delta
$$




$$
\begin{aligned}
& \leqq \sqrt{2}\left(\frac{k}{\frac{\varepsilon k}{2 N \operatorname{dim} P} N}+1\right) \delta \\
& =\sqrt{2}\left(\frac{2 \operatorname{dim} P}{\varepsilon}+1\right) \delta \\
& <\varepsilon,
\end{aligned}
$$

if $\frac{\varepsilon k}{N \operatorname{dim} P} \geqq 1$, so that $\left[\frac{\varepsilon k}{N \operatorname{dim} P}\right] \geqq 1$, and

$$
\delta<\frac{\varepsilon}{\sqrt{2}\left(\frac{2 \operatorname{dim} P}{\varepsilon}+1\right)} .
$$

Thus choosing $\delta$ sufficiently small and then

$$
k_{0}=\left[1+\frac{N \operatorname{dim} P}{\varepsilon}\right] \text {, }
$$

it follows that $Q_{k}$ chosen as above satisfies the conditions of the lemma.

Before we show that the singular part does not affect the entropy, we digress for a moment to show that if $U$ has singular spectrum the entropy of $\alpha_{U}$ taken with respect to any state $\varphi$ such that $\varphi \circ \alpha_{U}=\varphi$, is zero. In addition to being a result of more general type its proof will make the proof of the lemma following it more transparent.

Theorem 5.2. Let $U$ be a unitary operator on $H$ with spectral measure singular with respect to the Lebesgue measure. Let $\varphi$ be a state on $\mathscr{A}(H)$ such that $\alpha_{U}$ is $\varphi$-invariant. Then $h_{\varphi}\left(\alpha_{U}\right)=0$.

Proof. Let $P$ be an orthogonal projection in $B(H)$ of finite rank. Let $j: P(H) \rightarrow H$ be the inclusion map. Then there are, see [5] unital completely positive maps

$$
\begin{aligned}
& \alpha_{j}: \mathscr{A}(P(H)) \rightarrow \mathscr{A}(H) \quad \text { with } \quad \alpha_{j}(a(f))=a(j f), \\
& \alpha_{p}: \mathscr{A}(H) \rightarrow \mathscr{A}(P(H)) \quad \text { with } \quad \alpha_{p}(a(f))=a(p f) .
\end{aligned}
$$

If $P_{n} \nearrow 1$ is a sequence of such projections then with $j_{n}: P_{n}(H) \rightarrow H$ the inclusion,

$$
\alpha_{j_{n}} \circ \alpha_{p_{n}} \rightarrow \mathrm{id}_{\mathscr{A}(H)}
$$

in the pointwise-norm topology. By [3, Theorem V.2]

$$
h_{\varphi}\left(\alpha_{U}\right)=\lim _{n} h_{\varphi, \alpha_{U}}\left(\alpha_{j_{n}}\right)
$$

where

$$
h_{\varphi, \alpha_{U}}\left(\alpha_{j}\right)=\lim _{k \rightarrow \infty} \frac{1}{k} H_{\varphi}\left(\alpha_{j}, \alpha_{U} \alpha_{j}, \ldots, \alpha_{U}^{k-1} \alpha_{j}\right) .
$$

Whence it suffices to show

$$
h_{\varphi, \alpha_{U}}\left(\alpha_{j}\right)=0
$$


Let $P$ be as above. Since $\operatorname{dim} P<\infty$, given $\delta>0$ there is $\eta>0$ such that if $W_{1}, W_{2}: P(H) \rightarrow H$ are isometries with $\left\|W_{1}-W_{2}\right\|<\eta$, then, see [5]

$$
\left\|\alpha_{W_{1}}-\alpha_{W_{2}}\right\|<\delta
$$

where $\alpha_{W}(a(f))=a(W f)$. Let $Q_{k}$ be as in Lemma 5.1. Denote by pol $\left(Q_{k} U^{s} \mid P(H)\right)$ the partial isometry $W_{2}$ appearing in the polar decomposition

$$
Q_{k} U^{s}\left|P(H)=W_{2}\right|\left(Q_{k} U^{s} \mid P(H)\right) \mid .
$$

Let $W_{1}=U^{s} \mid P(H)$. Since

$$
\left\|U^{s} P-Q_{k} U^{s} P\right\|<\varepsilon \quad \text { if } \quad 0 \leqq s \leqq k,
$$

we can easily infer

$$
\left\|U^{s} \mid P(H)-\operatorname{pol}\left(Q_{k} U^{s} \mid P(H)\right)\right\| \leqq 3 \varepsilon
$$

if $\varepsilon \leqq \frac{1}{2}$, which we shall assume. Thus, choosing $\varepsilon<\frac{\eta}{3}$ we have for $k \geqq k_{0}$,

$$
\left\|\alpha_{U^{s} \mid P(H)}-\alpha_{\mathrm{pol}\left(Q_{k} U^{s} \mid P(H)\right)}\right\|<\delta \text { for } 0 \leqq s \leqq k .
$$

By [3, Proposition IV.3] there is for given $\chi>0$ and $\varepsilon>0, k_{0} \in \mathbb{N}$ such that if $k \geqq k_{0}$ and $Q_{k}$ are as in Lemma 5.1 then

$$
H_{\varphi}\left(\alpha_{j}, \alpha_{U} \alpha_{j}, \ldots, \alpha_{U}^{k-1} \alpha_{j}\right) \leqq k \chi+H_{\varphi}\left(\alpha_{\mathrm{pol}\left(Q_{k} j\right)}, \ldots, \alpha_{\mathrm{pol}\left(Q_{k} U^{k-1} j\right)}\right) .
$$

If we let $v: Q_{k}(H) \rightarrow H$ be the inclusion map then

$$
\alpha_{\text {pol }\left(Q_{k} j\right)}=\alpha_{v} \circ \alpha_{\text {pol }\left(Q_{k} j\right)},
$$

whence by [3, Proposition III.6(a) and 6(c)]

$$
H_{\varphi}\left(\alpha_{\mathrm{pol}\left(Q_{k} j\right)}, \ldots, \alpha_{\mathrm{pol}\left(Q_{k} U^{k-1} j\right)}\right) \leqq H_{\varphi}\left(\alpha_{v}, \ldots, \alpha_{v}\right)=H_{\varphi}\left(\alpha_{v}\right) .
$$

On the other hand by $[3$, III.4]

$$
H_{\varphi}\left(\alpha_{v}\right)=S\left(\varphi \circ \alpha_{v}\right)
$$

where $\varphi \circ \alpha_{v}$ is a state on $\mathscr{A}\left(Q_{k}(H)\right)$, a $C^{*}$-algebra of dimension less than $2^{k \varepsilon}$. Thus

$$
H_{\varphi}\left(\alpha_{v}\right) \leqq \log 2^{k \varepsilon}=k \varepsilon \log 2 .
$$

Hence by (1) and (2)

$$
\frac{1}{k} H_{\varphi}\left(\alpha_{j}, \alpha_{U} \alpha_{j}, \ldots, \alpha_{U}^{k-1} \alpha_{j}\right) \leqq \chi+\varepsilon \log 2 .
$$

Since $\chi$ and $\varepsilon$ are arbitrary, $h_{\varphi, \alpha v}\left(\alpha_{j}\right)=0$.

Lemma 5.3. Let $U$ be a unitary operator on $H$ with absolutely continuous part $U_{a}$ acting on $H_{a}$ and singular part $U_{s}$ acting on $H_{s}$. Let $A=A_{a} \oplus A_{s}$ commute with $U=U_{a} \oplus U_{s}, 0 \leqq A \leqq 1$. Assume $A_{a}$ and $U_{a}$ are as in Lemma 4.6. Then $h_{\omega_{A}}\left(\alpha_{U}\right)=$ $h_{\omega_{A_{a}}}\left(\alpha_{U_{a}}\right)$ is given by the formula in Lemma 4.6. Furthermore the same hold for the restrictions to $\mathscr{A}(H)_{e}$.

Proof. As in the proof of Lemma 4.6 we may restrict attention to the case when the spectrum of $U_{a}$ consists of a finite number of disjoint arcs. Furthermore if the 
multiplicity of $U_{a}$ on one of the arcs is infinite then both sides of the formula are infinite, hence we may assume each multiplicity is finite.

If we can prove the lemma for $U_{a}$ and $A_{a}$ as in Lemma 4.5 the general case follows from that case just as Lemma 4.6 followed from Lemma 4.5. So we assume $U_{a}=\bigoplus_{i=1}^{r} U_{i}$ with $U_{i}^{q}=V^{p_{2}}$, where $V$ is bilateral shift of multiplicity 1 , and $A_{a}=\bigoplus_{i=1}^{r} c_{i} 1_{i}, c_{i} \in[0,1]$. Let $X$ be as in the proof of Lemma 4.5. Thus $X$ has an orthonormal basis

$$
\left\{f_{11}, \ldots, f_{1 p_{1}}, \ldots, f_{r 1}, \ldots, f_{r p_{r}}\right\}
$$

where $f_{i k} \in H_{i}, V f_{i k}=f_{i(k+1)}$. To simplify notation let $W=U^{q}$, so that

$$
W=W_{a} \oplus W_{s}, \quad W_{a}=U_{a}^{q}=\bigoplus_{i=1}^{r} V^{p_{i}}, \quad W_{s}=U_{s}^{q} .
$$

For $n \in \mathbb{N}$ let

$$
X_{n}=\bigvee_{j=-n}^{n} W_{a}^{j} X
$$

Then $\bigcup_{n=0}^{\infty} X_{n}$ is dense in $H_{a}$. Choose an increasing sequence $\left(Y_{n}\right)$ of finite dimensional subspaces of $H_{s}$ with union dense in $H_{s}$. Then $\bigcup_{n} X_{n} \oplus Y_{n}$ is dense in $H$, so by [3, Theorem VII.4]

$$
h_{\omega_{A}}\left(\alpha_{W}\right)=\lim _{n} h_{\omega_{A}, \alpha_{W}}\left(\mathscr{A}\left(X_{n} \oplus Y_{n}\right)\right) .
$$

We use notation similar to that used in the proof of Theorem 5.2. Let

$$
j_{X_{n}}: X_{n} \rightarrow H_{a}, \quad j_{Y_{n}}: Y_{n} \rightarrow H_{a}, \quad j_{n}=j_{X_{n}} \oplus j_{Y_{n}}: X_{n} \oplus Y_{n} \rightarrow H
$$

be the inclusion maps, and let

$$
\alpha_{J_{n}}: \mathscr{A}\left(X_{n}\right) \rightarrow \mathscr{A}\left(H_{a}\right) \subset \mathscr{A}(H)
$$

etc. be the inclusion maps of the corresponding algebras. Fix $n \in \mathbb{N}$ and let $P$ be the orthogonal projection onto $Y_{n}$. Let $\varepsilon>0$ and $k_{0} \in \mathbb{N}$, and $Q_{k}$ for $k \geqq k_{0}$ be as in Lemma 5.1. Then

$$
\left\|W_{a}^{m} j_{X_{n}} \oplus W_{s}^{m} j_{Y_{n}}-W_{a}^{m} j_{X_{n}} \oplus \operatorname{pol}\left(Q_{k} W_{s}^{m} j_{Y_{n}}\right)\right\|, \quad 1 \leqq m \leqq k
$$

is small, so we can by [3, Proposition IV.3] assume

$$
H_{\omega_{A}}\left(\alpha_{j_{n}}, \alpha_{W} \alpha_{j_{n}}, \ldots, \alpha_{W}^{k-1} \alpha_{j_{n}}\right) \leqq k \varepsilon+H_{\omega_{A}}\left(\alpha_{J_{X_{n}} \oplus \operatorname{pol}\left(Q_{k} j_{Y_{n}}\right)}, \ldots, \alpha_{W_{a}^{k-1}{ }_{J_{X_{n}}} \oplus \operatorname{pol}\left(Q_{k} W_{s}^{k-1} j_{Y_{n}}\right)}\right) .
$$

Let $v: Q_{k}\left(H_{s}\right) \rightarrow H_{s}$ be the inclusion, and let $i_{n}: X_{n} \rightarrow X_{n}$ be the identity map. Then we have

$$
\left.\alpha_{W_{a}^{m} X_{n} \oplus \operatorname{pol}\left(Q_{k} W_{s}^{m} j_{Y_{n}}\right)}=\alpha_{W_{a}^{m} j_{X_{n}} \oplus v} \circ \alpha_{i_{n} \oplus \operatorname{pol}\left(Q_{k} W_{s}^{m} J_{Y_{n}}\right.}\right)
$$

It follows from [3, Proposition III.6(a)] that

$$
\begin{aligned}
& H_{\omega_{A}}\left(\alpha_{J_{X_{n}} \oplus \operatorname{pol}\left(Q_{k} / Y_{n}\right.}, \ldots, \alpha_{W_{a}^{k-1} j_{X_{n}} \oplus \operatorname{pol}\left(Q_{k} W_{s}^{k-1} j_{Y_{n}}\right)}\right) \\
& \quad \leqq H_{\omega_{A}}\left(\alpha_{j_{X_{n}} \oplus v}, \ldots, \alpha_{W_{a}^{k-1} j_{X_{n}} \oplus v}\right) \\
& \quad=H_{\omega_{A}}\left(\alpha_{J_{X_{n}} \oplus v}, \alpha_{W_{a} \oplus 1_{s}}{ }^{\circ} \alpha_{j_{X_{n}} \oplus v}, \ldots, \alpha_{W_{a} \oplus 1_{s}}^{k-1} \circ \alpha_{j_{X_{n}} \oplus v}\right),
\end{aligned}
$$


where $1_{s}$ is the identity on $H_{s}$. We may as in [3] identity $\alpha_{J_{X_{n}} \oplus v}$ with $M_{n}=\mathscr{A}\left(X_{n} \oplus\right.$ $\left.Q_{k}\left(H_{s}\right)\right)$. Then the last expression in the above inequality becomes

$$
H_{\omega_{A}}\left(M_{n}, \alpha_{W_{a} \oplus 1_{s}}\left(M_{n}\right), \ldots, \alpha_{W_{a} \oplus 1_{s}}^{k-1}\left(M_{n}\right)\right) .
$$

Let $Z=\bigoplus_{j=1}^{k-1} W_{a}^{j} X_{n}$. We may assume $Z$ has even dimension, so $\mathscr{A}(Z)$ is a factor. Then $Z$ has an orthonormal basis of eigenvectors for $A$, so $\omega_{A}$ factors between $\mathscr{A}(Z)$ and its relative commutant $\mathscr{A}(Z)^{\mathrm{c}}$ in $\mathscr{A}(H)$. Let $M=\mathscr{A}\left(Z \oplus Q_{k}\left(H_{\mathrm{s}}\right)\right)$. Then $\mathscr{A}(Z)^{c} \cap M \cong \mathscr{A}\left(Q_{k}\left(H_{s}\right)\right)$. Since also $\mathscr{A}(Z)$ is the tensor product with $\omega_{A}$ a product state of $2 n+k$ copies of $\mathscr{A}(X)$ we have, since $\operatorname{dim} \mathscr{A}\left(Q_{k}\left(H_{s}\right)\right) \leqq 2^{k \varepsilon}$,

$$
\begin{aligned}
S\left(\omega_{A} \mid M\right) & =(2 n+k) S\left(\omega_{A} \mid \mathscr{A}(X)\right)+S\left(\omega_{A} \mid \mathscr{A}(Z)^{c} \cap M\right) \\
& \leqq(2 n+k) S\left(\omega_{A} \mid \mathscr{A}(X)\right)+k \varepsilon \log 2 .
\end{aligned}
$$

Since $M$ contains the algebra generated by $M_{n}, \alpha_{W_{a} \oplus 1_{s}}\left(M_{n}\right), \ldots, \alpha_{W_{a} \oplus 1_{s}}^{k-1}\left(M_{n}\right)$, it follows from [3, Lemma VIII.1] that

$$
H_{\omega_{A}}\left(M_{n}, \alpha_{W_{a} \oplus 1_{s}}\left(M_{n}\right), \ldots, \alpha_{W_{a} \oplus 1_{s}}^{k-1}\left(M_{n}\right)\right) \leqq(2 n+k) S\left(\omega_{A} \mid \mathscr{A}(X)\right)+k \varepsilon \log 2 .
$$

We thus have, going back in the proof

$$
\frac{1}{k} H_{\omega_{A}}\left(\alpha_{j_{n}}, \alpha_{W} \alpha_{j_{n}}, \ldots, \alpha_{W}^{k-1} \alpha_{j_{n}}\right) \leqq \varepsilon+\frac{2 n+k}{k} S\left(\omega_{A} \mid \mathscr{A}(X)\right)+\varepsilon \log 2
$$

We therefore conclude that

$$
h_{\omega_{A}, \alpha_{W}}\left(\alpha_{j_{n}}\right) \leqq S\left(\omega_{A} \mid \mathscr{A}(X)\right),
$$

whence

$$
h_{\omega_{A}}\left(\alpha_{W}\right) \leqq S\left(\omega_{A} \mid \mathscr{A}(X)\right)
$$

and therefore

$$
h_{\omega_{A}}\left(\alpha_{U}\right)=\frac{1}{q} h_{\omega_{A}}\left(\alpha_{W}\right) \leqq \frac{1}{q} S\left(\omega_{A} \mid \mathscr{A}(X)\right) .
$$

Now from the proof of Lemma 4.5 we have

$$
h_{\omega_{A_{a}}}\left(\alpha_{U_{a}}\right)=\frac{1}{q} S\left(\omega_{A_{a}} \mid \mathscr{A}(X)\right) .
$$

By Lemma $4.1 h_{\omega_{A_{a}}}\left(\alpha_{U_{a}}\right) \leqq h_{\omega_{A}}\left(\alpha_{U}\right)$, hence they are equal, completing the proof of the lemma for $h_{\omega_{A}}\left(\alpha_{U}\right)$.

To see that the entropy is the same for the restriction to the even algebra $\mathscr{A}(H)_{e}$ we know by Lemmas 3.1 and 4.1 and the first part that

$$
\begin{aligned}
h_{\omega_{A_{a}} \mid \mathscr{A}(H)_{e}}\left(\alpha_{U_{a}} \mid \mathscr{A}(H)_{e}\right) & \leqq h_{\omega_{A} \mid \mathscr{A}(H)_{e}}\left(\alpha_{U} \mid \mathscr{A}(H)_{e}\right) \\
& \leqq h_{\omega_{A}}\left(\alpha_{U}\right) \\
& =h_{\omega_{A_{a}}}\left(\alpha_{U_{a}}\right) .
\end{aligned}
$$

But by Lemma $4.6 h_{\omega_{A_{a}} \mid \mathscr{A}(H)_{e}}\left(\alpha_{U_{a}} \mid \mathscr{A}(H)_{e}\right)=h_{\omega_{A_{a}}}\left(\alpha_{U_{a}}\right)$, completing the proof for the restrictions to the even algebra. 


\section{The Entropy Formula}

We prove two formulas. When the quasifree state is of the form $\omega_{\lambda}$ we first express the entropy by the multiplicity function of $U$. Then we prove the general formula when $A$ has pure point spectrum. Recall that we use the notation $m(U)$ to denote the multiplicity function of the absolutely continuous part $U_{a}$ of $U$. To express the formulas we use direct integral theory as described in the introduction based on $U_{a}$.

Theorem 6.1. With $U$ a unitary operator on the Hilbert space $H$ and $0 \leqq \lambda \leqq 1$, we have

$$
h_{\omega_{\lambda}}\left(\alpha_{U}\right)=\frac{1}{2 \pi}(\eta(\lambda)+\eta(1-\lambda)) \int_{0}^{2 \pi} m(U(\theta)) d \theta .
$$

Proof. If $\lambda=0$ or 1 then $\omega_{\lambda}$ is a pure state, see e.g. [8], so $h_{\omega_{\lambda}}\left(\alpha_{U}\right)=0$ by Lemma 3.1. Since $\eta(\lambda)+\eta(1-\lambda)=0$ when $\lambda \in\{0,1\}$ the formula holds in this case. Assume $0<\lambda<1$. Since by [3, VII.5] $h_{\omega_{\lambda}}\left(\alpha_{U}^{n}\right)=|n| h_{\omega_{\lambda}}\left(\alpha_{U}\right)$, it follows from Lemmas 4.3, 4.4 and 4.5 that all the conditions of Theorem 2.1 are satisfied. Thus the formula holds when $U$ has Lebesgue spectrum.

Let now $U=U_{a} \oplus U_{s}$ be the decomposition of $U$ into absolutely continuous and singular parts acting on $H_{a}$ and $H_{s}$ respectively. Let $\varepsilon>0$ be given. By measure theory there is a unitary $V$ with Lebesgue spectrum on $H_{a}$ such that its multiplicity function satisfies

$$
m(V)=\sum_{j=1}^{r} d_{j} \chi_{X} \geqq m(U),
$$

where $X_{j}$ is an arc of the form $\exp \left(2 \pi i\left[a_{i}, b_{i}\right]\right)$ with $b_{i}-a_{i}$ rational, and

$$
\int_{0}^{2 \pi} m(U(\theta)) d \theta+\varepsilon>\int_{0}^{2 \pi} m(V(\theta)) d \theta .
$$

We then have, by the first paragraph of the proof and Lemmas 4.1, 4.3, 5.3 in that order

$$
\begin{aligned}
\frac{1}{2 \pi}(\eta(\lambda)+\eta(1-\lambda)) \int_{0}^{2 \pi} m(U(\theta)) d \theta & =h_{\omega_{\lambda}}\left(\alpha_{U_{a}}\right) \\
& \leqq h_{\omega_{\lambda}}\left(\alpha_{U_{a} \oplus U_{s}}\right) \\
& \leqq h_{\omega_{\lambda}}\left(\alpha_{V \oplus U_{s}}\right) \\
& =h_{\omega_{\lambda}}\left(\alpha_{V}\right) \\
& =\frac{1}{2 \pi}(\eta(\lambda)+\eta(1-\lambda)) \int_{0}^{2 \pi} m(V(\theta)) d \theta \\
& <\frac{1}{2 \pi}(\eta(\lambda)+\eta(1-\lambda))\left[\int_{0}^{2 \pi} m(U(\theta)) d \theta+\varepsilon\right] .
\end{aligned}
$$

Since $\varepsilon$ is arbitrary the formula follows.

We use direct integral as described in the introduction. If $A$ commutes with $U=U_{a} \oplus U_{s}$ then $A=A_{a} \oplus A_{s}$. We have

$$
A_{a}=\int_{\mathbf{T}}^{\oplus} A(\theta) d \theta
$$


with $A(\theta) \in B\left(H_{\theta}\right)$, where $H_{\theta}=0$ if $m(U(\theta))=0$. Suppose $A$ has pure point spectrum, so that

$$
A=\sum_{j \in J} \lambda_{j} e_{j}
$$

with $J$ finite or countably infinite, and $\left(e_{j}\right)$ as an orthogonal family of projections with $\operatorname{sum} 1$, and $0 \leqq \lambda_{j} \leqq 1$. Denote by

$$
U_{j}=U \mid e_{j}(H) .
$$

We denote by $\operatorname{Tr}$ the usual trace on $B\left(H_{\theta}\right)$. Writing $e_{j}=\int{ }^{\oplus} e_{j}(\theta) d \theta$, and $c(\lambda)=\eta(\lambda)+$ $\eta(1-\lambda)$ we have

$$
\operatorname{Tr}(c(A(\theta)))=\sum c\left(\lambda_{j}\right) \operatorname{Tr}\left(e_{j}(\theta)\right)=\sum c\left(\lambda_{j}\right) m\left(U_{j}(\theta)\right) .
$$

Thus the following lemma is immediate from Theorem 6.1.

Lemma 6.2. With the above notation and assumptions we have

$$
\sum_{j \in J} h_{\omega_{\lambda_{j}}}\left(\alpha_{U_{j}}\right)=\frac{1}{2 \pi} \int_{0}^{2 \pi} \operatorname{Tr}(\eta(A(\theta))+\eta(1-A(\theta))) d \theta .
$$

Theorem 6.3. Let $0 \leqq A \leqq 1$ be an operator with pure point spectrum acting on $H$. Let $U$ be a unitary operator on $H$ commuting with $A$. Then we have

$$
h_{\omega_{A}}\left(\alpha_{U}\right)=\frac{1}{2 \pi} \int_{0}^{2 \pi} \operatorname{Tr}(\eta(A(\theta))+\eta(1-A(\theta))) d \theta .
$$

Proof. If $A=\sum_{i=1}^{\infty} \lambda_{i} e_{i}$ the projections $p_{n}=\sum_{i=1}^{n} e_{i}$ define expectations on $\mathscr{A}(H)$ satisfying the conditions of Lemma 3.3. Hence to show the formula we may assume $A$ has finite spectrum.

By Lemma 6.2 it suffices to show

$$
h_{\omega_{A}}\left(\alpha_{U}\right)=\sum_{j \in J} h_{\omega_{\lambda_{j}}}\left(\alpha_{U_{j}}\right)
$$

where $A=\sum_{j \in J} \lambda_{j} e_{j}, J$ finite. Let $\mathscr{A}(H)_{e}$ be the even algebra. An inspection of the proof of Theorem 6.1 shows that the results used in the proof all hold for $\omega_{\lambda} \mid \mathscr{A}(H)_{e}$ and $\alpha_{U} \mid \mathscr{A}(H)_{e}$. We thus have

$$
h_{\omega_{\lambda}}\left(\alpha_{U} \mid \mathscr{A}(H)_{e}\right)=h_{\omega_{\lambda}}\left(\alpha_{U}\right)
$$

We therefore have, using Lemma 3.2 and Remark 4.2 together with Lemma 3.4,

$$
\begin{aligned}
h_{\omega_{A}}\left(\alpha_{U}\right) & \geqq h_{\omega_{A}}\left(\alpha_{U} \mid \mathscr{A}(H)_{e}\right) \\
& \geqq \sum_{j \in J} h_{\omega_{\lambda_{J}}}\left(\alpha_{U} \mid \mathscr{A}\left(e_{j} H\right)_{e}\right) \\
& =\sum_{j \in J} h_{\omega_{\lambda_{j}}}\left(\alpha_{U_{J}}\right) .
\end{aligned}
$$

It therefore remains to show the converse inequality. For this we may assume the absolutely continuous part $U_{a}$ of $U$ acts as a multiplication operator on a subset $X$ of the circle $\mathbf{T}$, and that $H_{a}=L^{2}(X, d \theta)$ considered as a subspace of 
$L^{2}(\mathbf{T}, d \theta)$. We first consider the case when $m(U)$ is bounded. Thus

$$
m(U)=\sum_{n=1}^{N} n \chi_{X_{n}},
$$

where $X_{n}=m(U)^{-1}(n)$. Since $A$ has finite spectrum we can subdivide each $X_{n}$ into a disjoint union of Borel sets $X_{n r}$ on which $A(\theta)$ is constant, i.e. has same finite spectrum counted with multiplicity. Thus we have

$$
A_{a}=\bigoplus_{n, r} \int_{X_{n r}}^{\oplus} A(\theta) d \theta=\bigoplus_{n, r} A_{n r} \otimes 1_{n r},
$$

where $1_{n r}$ is the identity on $L^{2}\left(X_{n r}, d \theta\right)$.

Let $\varepsilon>0$ be given. Choose $Y_{n r} \subset X_{n r}$ a closed set, and choose open sets $O_{n r} \supset Y_{n r}$ which are disjoint and with each $O_{n r}$ a finite union of arcs of the form $\exp \left(2 \pi i\left[a_{i}, b_{i}\right]\right)$ with $b_{j}-a_{j}$ rational and the size of $O_{n r}$ to be determined below.

Let $P_{n r}$ be the orthogonal projection of $L^{2}(\mathbf{T}, d \theta)$ onto $L^{2}\left(Y_{n r}, d \theta\right)$, and let

$$
e=\bigoplus_{n r} P_{n r} \text {. }
$$

Let $V_{a}$ be the unitary multiplication operator on $H_{1}=L^{2}\left(\bigcup_{n, r} O_{n r}, d \theta\right)$ with multiplicity function

$$
m\left(V_{a}\right)=\sum_{n, r} n \chi_{o_{n r}}
$$

and let

$$
V=V_{a} \oplus U_{s}
$$

Define $\tilde{A}$ by $\tilde{A}=\tilde{A}_{a} \oplus A_{s}$, where

$$
\tilde{A}_{a}=\bigoplus_{n r} A_{n r} \otimes 1_{o_{n r}},
$$

where $1_{O_{n r}}$ is the identity on $L^{2}\left(O_{n r}, d \theta\right)$. Then we have $\tilde{A} e=A e$ and $V e=U e$, and furthermore by Theorem 6.1 and Lemma 6.2

$$
h_{\omega_{\bar{A}}}\left(\alpha_{V}\right)=\sum h_{\omega_{\lambda}}\left(V_{j}\right)
$$

where $V_{j}=V \mid e_{j}(H)$, since $V$ and $\tilde{A}$ satisfy the assumptions of Lemma 4.6.

We now make our choice of the size of the $O_{n r}$ 's, namely we choose them so close to the $Y_{n r}$ 's that

$$
h_{\omega_{\lambda_{j}}}\left(\alpha_{V_{j}}\right)<h_{\omega_{\lambda,}}\left(\alpha_{(U e)_{j}}\right)+\varepsilon 2^{-j}
$$

where $(U e)_{j}=U e \mid e_{j}(H)$. This can be done since $A$ has finite spectrum, $U e=V e$, and the theorem is true for $V$. We thus have

$$
\begin{aligned}
h_{\omega_{A}}\left(\alpha_{U e \oplus U_{s}}\right) & =h_{\omega_{A}}\left(\alpha_{V_{a} e \oplus U_{s}}\right) & & \\
& \leqq h_{\omega_{A}}\left(\alpha_{V_{a} \oplus U_{s}}\right) & & \text { (by Lemma 4.1) } \\
& =h_{\omega_{\tilde{A}_{a}}}\left(\alpha_{V_{a}}\right) & & \text { (by Lemma 5.3) } \\
& =\sum_{j} h_{\omega_{\lambda_{j}}}\left(\alpha_{V_{J}}\right) & & \text { (by Lemma 6.2) }
\end{aligned}
$$




$$
\begin{aligned}
& <\sum_{j}\left(h_{\omega_{\lambda_{j}}}\left(\alpha_{U e)_{J}}\right)+\varepsilon 2^{-j}\right) \\
& \leqq \sum_{j} h_{\omega_{\lambda_{j}}}\left(\alpha_{U_{J}}\right)+\varepsilon
\end{aligned}
$$

This holds for all $\varepsilon>0$, so we conclude

$$
h_{\omega_{A}}\left(\alpha_{U e \oplus U_{s}}\right) \leqq \sum_{j} h_{\omega_{\lambda_{j}}}\left(\alpha_{U_{J}}\right) \text {. }
$$

The projections $e$ can be constructed to form an increasing sequence when $\varepsilon \searrow 0$. Since each $e$ commutes with $A$ it defines an $\omega_{A}$ invariant expectation on $\mathscr{A}(H)$, so by Lemma 3.3 we have

$$
h_{\omega_{A}}\left(\alpha_{U}\right)=\lim _{\varepsilon \rightarrow 0} h_{\omega_{A}}\left(\alpha_{U e \oplus U_{s}}\right),
$$

hence we have $h_{\omega_{A}}\left(\alpha_{U}\right) \leqq \sum_{j} h_{\omega_{\lambda_{j}}}\left(\alpha_{U}\right)$, as we wanted to show, proving the theorem when $U_{a}$ has bounded spectrum.

In the general case let $P_{N}$ be the projection of $H_{a}$ onto the spectral subspace where $m(U) \leqq N$. Then $P_{N} \nearrow 1$ as $N \rightarrow \infty$. Since the theorem holds for $\bigcup P_{N}$ by the first part of the proof it follows again from Lemma 3.3 that it holds for $U$.

Acknowledgements. The authors are happy to express their gratitude to Institut Mittag Leffler for making it possible for them to spend an extensive time working together on this work. Thanks go also to IHES, University of New South Wales, University of California, Berkeley and UCLA, where the authors' visits proved beneficial for this work. Special thanks go to A. Connes for suggesting the problem and conjecturing (1).

\section{References}

1. Besson, O.: The entropy of quantum Markov states. Lecture Notes in Mathematics vol 1136, pp. 81-89. Berlin, Heidelberg, New York: Springer 1985

2. Choda, M.: Entropy for *-endomorphisms and relative entropy for subalgebras (to appear)

3. Connes, A., Narnhofer, H. Thirring, W.: Dynamical entropy of $C^{*}$-algebras and von Neumann algebras. Commun. Math. Phys. 112, 691-719 (1987)

4. Connes, A., St $\phi$ rmer, E.: Entropy of automorphisms of $\mathrm{II}_{1}$ von Neumann algebras. Acta. Math. 134, 289-306 (1975)

5. Evans, D.: Completely positive quasifree maps on the CAR-algebra. Commun. Math. Phys. 70, 53-68 (1979)

6. Kosaki, M.: Interpolation theory and the Wigner-Yanase-Dyson-Lieb concavity. Commun. Math. Phys. 87, 315-329 (1982)

7. Pimser, M., Popa, S.: Entropy and index for subfactors. Ann. Scient. Éc. Norm. Sup. 19, 57-106 (1986)

8. Powers, R. T., St $\phi$ rmer, E.: Free states of the canonical anticommutation relations. Commun. Math. Phys. 16, 1-33 (1970)

9. Pusz, W., Woronowicz, S.: Form convex functions and the WYDL and other inequalities. Lett. Math. Phys. 2, 505-512 (1978) 